\title{
Evaluation of the Input Impedance and Impedance Quality Factor of a Dipole in Spatial and Spectral Domains
}

\author{
Michal CERVENY, Pavel HAZDRA \\ Dept. of Electromagnetic Field, FEE, Czech Technical University, Technicka 2, 16627 Prague, Czech Republic \\ cervemi5@fel.cvut.cz, hazdrap@fel.cvut.cz \\ Submitted May 5, 2017 / Accepted August 7, 2017
}

\begin{abstract}
In classic antenna analysis, there exist several methods of calculating input impedance when the source distribution of an antenna is known. The well-known induced EMF method, based on spatially distributed currents, is typically applied for this purpose and, thereby, serves as a reference for this research. Conversely, the spectral domain method takes the opposite approach for the input impedance and is evaluated using a far-field pattern. This paper compares the induced EMF method and the spectral domain method. Furthermore, the quality factor of the dipole antenna, based on the impedance variation with frequency, is calculated for both methods. This study shows that the induced EMF method and the spectral domain method are in close agreement.
\end{abstract}

\section{Keywords}

Dipole, input impedance, quality factor, spatial domain, spectral domain, EMF method

\section{Introduction}

Quality factor $Q$ [1] is one of the most fundamental characteristics of an antenna because of its connection to a bandwidth [2]. There are many ways to evaluate quality factor $Q$, both in the time [3] and frequency domains [4].

The most popular method is probably the one based on input impedance frequency changes [1], [4]. If the distribution of currents on an antenna is known, the input impedance, and, consequently, quality factor $Q$ [4], can be evaluated by the complex power balance in the spatial domain. This approach is known as the induced EMF method or the Poynting vector method [5].

Another way to determine $Q$ is by spectral analysis, which is, in turn, based on the integration of the radiation pattern in visible and invisible space [6-8]. Integration in visible space (over real angles), which is well-known and demonstrated in the literature [5], facilitates the classic calculation of the radiation resistance of an antenna. A less well-known fact is that extending the integration to invisible space (complex angles) also allows the information pertaining to the reactance to become available thanks to the special properties of the radiation pattern as explained in [6].

In this paper we study the quality factor $Q$ of a thin dipole from its input impedance by both methods, i.e., in the spatial and spectral domain method. The source current is prescribed as sinusoidal (one-term approximation which permits the semi-analytical evaluation of the background integrals).

\section{The Induced EMF Method}

When considering a thin linear antenna oriented in the $\mathrm{z}$-axis, the input impedance (referred to current maximum) is given by the following double spatial integral [9]:

$Z=$

$\frac{\mathrm{j} 30}{k\left|I_{0}\right|^{2}} \iint_{z z^{\prime}}\left[k^{2} I(z) \cdot I\left(z^{\prime}\right)-\frac{\partial}{\partial z} I(z) \frac{\partial}{\partial z^{\prime}} I\left(z^{\prime}\right)\right] \frac{\mathrm{e}^{-\mathrm{j} k R}}{R} \mathrm{~d} z \mathrm{~d} z^{\prime}$

where the current is assumed as:

$$
I=I_{0} \sin \left[k\left(\frac{L}{2}-|z|\right)\right]
$$

where $k$ is wavenumber and $L$ is dipole length respectively. The wire radius $a$ is incorporated in the thin-wire kernel by

$$
R=\sqrt{\left(z-z^{\prime}\right)^{2}+a^{2}} .
$$

The result of (1) is available in closed form [5] and is used as a reference.

\section{Spectral Domain Method}

The spectral domain approach considers a z-oriented thin-strip dipole of length $L$ and width $w$. However, it was shown [7] that thin-strip and small-radius dipoles are electromagnetically equivalent when 


$$
a=\frac{w}{\mathrm{e}^{\frac{3}{2}}} \approx \frac{w}{4.48} .
$$

Hence, the above equation allows a comparison to be made between the thin-wire and thin-strip structures which is subsequently used to calculate the results.

The thin-strip current is assumed to be constant along the dipole width:

$$
I=\frac{I_{0}}{2 w} \sin \left[k\left(\frac{L}{2}-|z|\right)\right] .
$$

Following the approach from [7], the far-field pattern is a Fourier transform of (5):

$$
F=\frac{k Z_{0} I_{0}}{(2 \pi)^{2}} \frac{\sin \frac{k_{\mathrm{y}} w}{2}}{\frac{k_{\mathrm{y}} w}{2}} \frac{\cos \frac{k_{\mathrm{z}} L}{2}-\cos \frac{k L}{2}}{k^{2}-k_{\mathrm{z}}^{2}}
$$

where $k_{\mathrm{y}}$ and $k_{\mathrm{z}}$ are vector propagation constant components and $k^{2}=k_{\mathrm{y}}{ }^{2}+k_{\mathrm{z}}{ }^{2}$. Consequently, the real and imaginary parts of the input impedance are given by [6]:

$$
\begin{aligned}
& R=\frac{2 Z_{0} k}{\pi^{2}} \int_{0}^{k} \frac{\left(\cos \frac{k_{\mathrm{z}} L}{2}-\cos \frac{k L}{2}\right)^{2}}{k^{2}-k_{\mathrm{z}}^{2}} \\
& \cdot\left[\int_{0}^{\sqrt{k^{2}-k_{\mathrm{z}}^{2}}}\left(\frac{\sin \frac{k_{\mathrm{y}} w}{2}}{\frac{k_{\mathrm{y}} w}{2}}\right)^{2} \frac{\mathrm{d} k_{\mathrm{y}}}{\sqrt{\left(k^{2}-k_{\mathrm{z}}^{2}\right)-k_{\mathrm{y}}^{2}}}\right] \mathrm{d} k_{\mathrm{z}}, \\
& X=\frac{2 Z_{0} k}{\pi^{2}}\left\{\int_{0}^{k} \frac{\left(\cos \frac{k_{\mathrm{z}} L}{2}-\cos \frac{k L}{2}\right)^{2}}{k^{2}-k_{\mathrm{z}}^{2}}\right. \\
& {\left[\int_{\sqrt{k^{2}-k_{\mathrm{z}}^{2}}}^{\infty}\left(\frac{\sin \frac{k_{\mathrm{y}} w}{2}}{\frac{k_{\mathrm{y}} w}{2}}\right)^{2} \frac{\mathrm{d} k_{\mathrm{y}}}{\sqrt{k_{\mathrm{y}}^{2}-\left(k^{2}-k_{\mathrm{z}}^{2}\right)}}\right] \mathrm{d} k_{\mathrm{z}}} \\
& -\int_{k}^{\infty} \frac{\left(\cos \frac{k_{\mathrm{z}} L}{2}-\cos \frac{k L}{2}\right)^{2}}{k_{\mathrm{z}}^{2}-k^{2}} \\
& \left.\left[\int_{0}^{\infty}\left(\frac{\sin \frac{k_{\mathrm{y}} w}{2}}{\frac{k_{\mathrm{y}} w}{2}}\right)^{2} \frac{\mathrm{d} k_{\mathrm{y}}}{\sqrt{k_{\mathrm{y}}^{2}+\left(k_{\mathrm{z}}^{2}-k^{2}\right)}}\right] \mathrm{d} k_{\mathrm{z}}\right\} .
\end{aligned}
$$

The numerical evaluation of these integrals will be discussed in Sec. 5.

\section{Quality Factor}

For the purpose of this paper, we use a well-established definition of the quality factor based on the variation of the input impedance with frequency [1], [4]:

$$
Q=\frac{f}{2 R_{0}}\left|\frac{\partial Z}{\partial f}\right|_{f=f_{0}} \text {. }
$$

\section{Numerical Validation}

Equations (7-9) have been numerically evaluated in Matlab [10] using the rectangle method for integration. The input impedance and the untuned quality factor (9) of a dipole antenna have been investigated and compared with the analytical solution of (1).

Since equation (8) for the reactance contains the definite integrals with infinite upper limits, the numerical implementation is not straightforward making it necessary to estimate how large a number is needed to replace the infinite limits, and how many subintervals must be used to obtain a calculation with sufficient accuracy.

The initial value for the upper limits has been chosen $(N=20000)$ and the integrals have been calculated in 20000 subintervals. The input impedance of a strip dipole of length $L$, ranging from $0.15 \lambda$ to $0.6 \lambda$, with widths of $0.01 \lambda, 0.001 \lambda$ and $0.0001 \lambda$ of the dipole, are presented in Fig. 1 which depicts both the EMF method and the spectral domain method.

It can be seen that the input reactance for both methods, in particular the short dipole, is very similar for $w=0.01 \lambda$ and $0.001 \lambda$. The results for the longer lengths show greater variation. Furthermore, the radiation resistance $R$ (the spectral domain method) and $R_{\mathrm{EMF}}$ (the EMF method) provide the same results with only negligible differences as both methods overlap perfectly for all three widths in the graph.

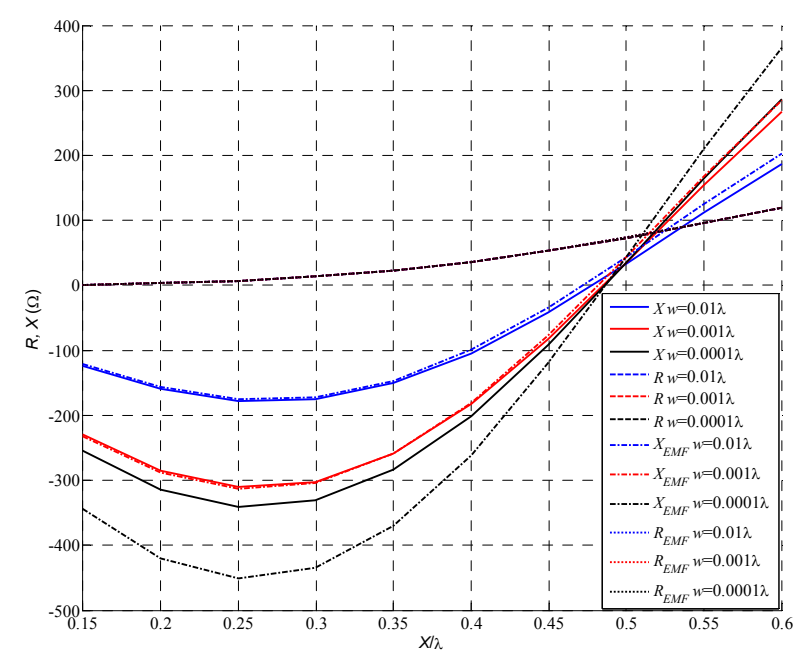

Fig. 1. Input impedance evaluated by (7), (8) of a dipole vs. electrical length for $N=20000$, compared with EMF. 
How the number of subintervals changes the results of the spectral domain method can be seen in the following scenario. A dipole antenna of width $0.001 \lambda$ and length $0.6 \lambda$ has been considered and the upper limit has been set again to $N=20000$. For 20000,50000 and 100000 subintervals the differences between $X_{\mathrm{EMF}}$ and $X$ are $16.5 \Omega$, $11.4 \Omega$ and $8.7 \Omega$, respectively. The expected trend shows that increasing the number of subintervals decreases the differences between the two methods. From the results it can also be seen that the convergence of the rectangle method, which was used for integration, is slow and the presented differences between the two methods are given mainly by this numerical error.

The effect of the low value of the upper limit can be seen in Fig. 1 where the shifted black solid line $(w=0.0001 \lambda)$ does not reach the dash-dotted EMF line.

To determine which upper limit of the integrals is suitable, the convergence of the spectral domain method has been investigated. Figure 2 clearly illustrates the differences between the EMF and spectral domain methods. The input reactance is shown for the width of $0.0001 \lambda$ and lengths $0.4,0.5$ and $0.6 \lambda$. The results are displayed for the upper limit $N$ from 5000 to 640000 . It can be seen that the input reactance requires the upper limit to be set to approximately 300000 in this case where the spectral domain method converges for all three widths of the dipole.

According to the previous results, the upper limits have been set to $N=300000$ and every integral has been calculated in 300000 subintervals. Now it can be seen that both methods agree well for the width of $0.0001 \lambda$, especially the short dipoles.

In the next step, the untuned quality factor $Q$ has been calculated for both methods using (9) as it is presented in Fig. 4. The width of the dipole antenna is $0.01 \lambda$, $N=20000$ and 10000 subintervals have been chosen for a faster but still reliable calculation as the graph is constructed from 60 different samples of $L / \lambda$. It can be seen

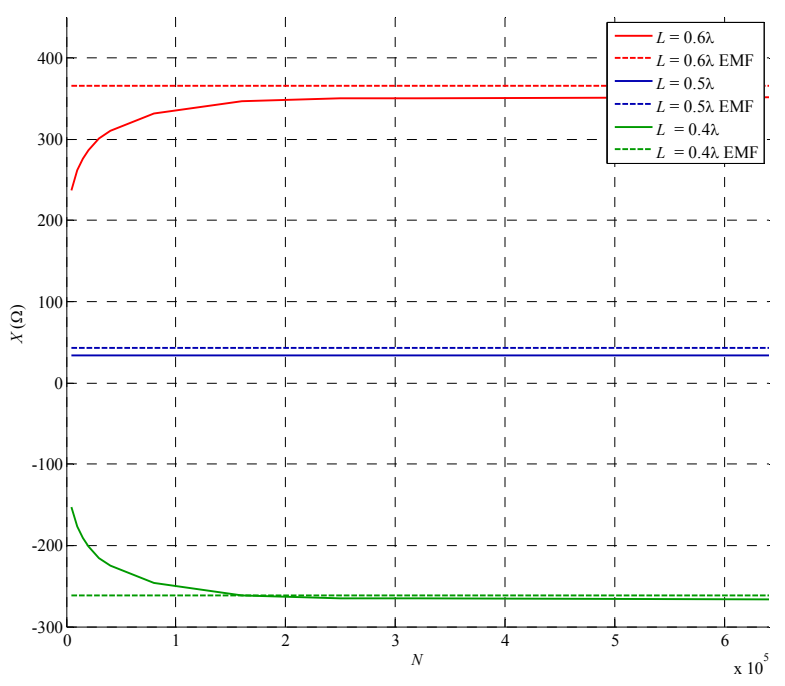

Fig. 2. Input reactance of dipoles of varying lengths with width $0.0001 \lambda$ versus integration limit $N$.

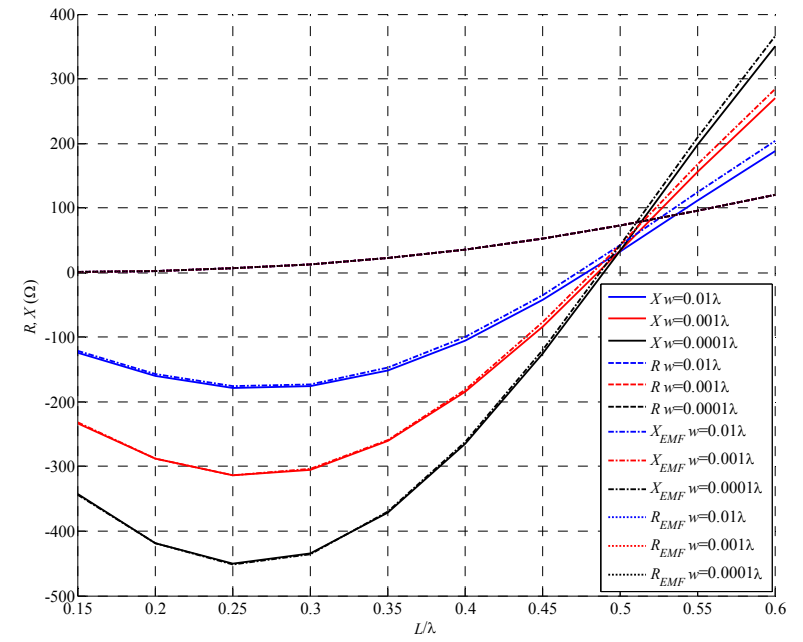

Fig. 3. Input impedance of a dipole versus electrical length, $N=300000$, compared with EMF.

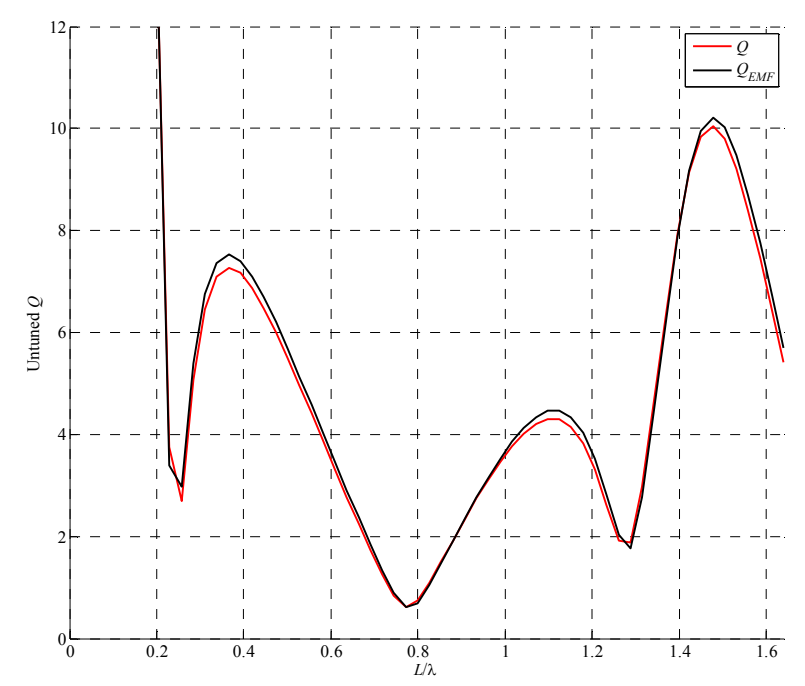

Fig. 4. Untuned quality factor of a dipole antenna of length $L$.

that both methods present a very similar pattern of quality factor $Q$.

The last graph (Fig. 5) shows the quality factor $Q$ of a dipole antenna $(L=0.5 \lambda)$ for both methods versus the width of the antenna. Moreover, the results of the closedform equation for radiation $Q$ are displayed for comparative purposes [11]. The well-known fact that wider dipole antennas have a lower quality factor can be seen in the figure. In this case, the same settings that were used in previous calculations have been used when calculating the $Q$ factor.

The differences between the results can be caused by several factors. The first is the previously mentioned numerical error in the integration where an increased number of subintervals is required and, unfortunately, it will cause a significant increase in the calculation time. Thus, future work could incorporate more advanced techniques for the numerical evaluation of integrals. Secondly, the Induced EMF method is only valid for very thin dipoles in terms of wavelength which is also true for the calculation of the equivalent radius in (8). 


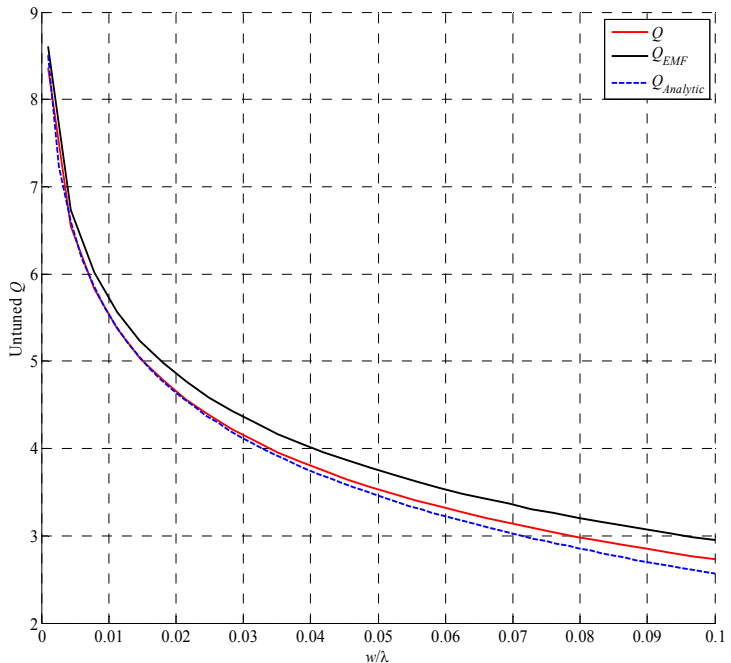

Fig. 5. Quality factor of a dipole antenna of width $w$.

\section{Conclusion}

In this paper we have studied two completely different methods for the calculation of the complex input impedance of a dipole antenna. The first one (EMF method) is based on spatially distributed currents on a thin dipole while the second one (spectral domain method) evaluates the input impedance from a far-field pattern obtained by the Fourier transform of the current's density. While the radiation resistance in the spectral domain method can be easily calculated with a classic numerical approach, the reactance requires more effort as the integrals with infinite upper limits need to be evaluated.

For the numerical calculation of the reactance, the infinite upper limits have been replaced by a finite number. From our graphs it can be seen that the number can be estimated via a convergence of the results. Furthermore, the number of subintervals can be estimated when the characteristics calculated by the EMF method are known. The disadvantage of this numerical approach lies in the calculation time, as it can take several days for a ubiquitous, generic PC to complete the calculations. In order to improve the accuracy and the computation time more advanced integration techniques are required.

In conclusion, the upper limit can be lower for thicker dipoles. When a calculation of a thin dipole is required, a higher upper limit has to be set. To maintain the accuracy of the results, the number of subintervals has to be increased appropriately, especially for dipoles longer than $0.4 \lambda$.

The quality factor of the dipole antenna has been calculated by both methods and the results are in close agreement.

Finally, from the application point of view, for its simplicity and closed-form, the induced EMF method is more convenient for determining dipole impedance and consequently, the quality factor.

\section{Acknowledgments}

This work has been supported by the project 17-00607S “Complex Artificial Electromagnetic Structures and Nanostructures".

\section{References}

[1] YAGHJIAN, A. D., BEST, S. R. Impedance, bandwidth and Q of antennas. IEEE Transactions on Antennas and Propagation, 2005, vol. 53, no. 4 , p. 1298-1324. DOI: 10.1109/TAP.2005.844443

[2] CAPEK, M., JELINEK, L., HAZDRA, P. On the functional relation between quality factor and fractional bandwidth. IEEE Transactions on Antennas and Propagation, 2015, vol. 63, no. 6, p. 2787-2790. DOI: 10.1109/TAP.2015.2414472

[3] CAPEK, M., JELINEK, L., VANDENBOSCH, G. A. E., HAZDRA, P. Time domain scheme for stored energy evaluation. In IEEE International Symp. on Antennas and Propagation \& USNC/URSI National Radio Science Meeting. Vancouver (Canada), 2015, p. 1706-1707. DOI: 10.1109/APS.2015.7305242

[4] CAPEK, M., JELINEK, L., HAZDRA, P., EICHLER, J. The measurable $\mathrm{Q}$ factor and observable energies of radiating structures. IEEE Transactions on Antennas and Propagation, 2014, vol. 62, no. 1, p. 311-318. DOI: 10.1109/TAP.2013.2287519

[5] JORDAN, C. Electromagnetic Waves and Radiating Systems. $2^{\text {nd }}$ edition, Prentice Hall, 1968. ISBN: 10: 0132499959

[6] RHODES, D. R. On a fundamental principle in the theory of planar antennas. Proceedings of the IEEE, 1964, vol. 52, no. 9, p. 1013-1021. DOI: 10.1109/PROC.1964.3231

[7] RHODES, D. R. On the stored energy of planar apertures. IEEE Transactions on Antennas and Propagation, 1966, vol. 14, no. 6, p. 676-683. DOI: 10.1109/TAP.1966.1138801

[8] TIWARI, A. K., PODDAR, D. R., DAS, B. N. On the equivalent radius of a radiating slot in impedance calculations. Progress In Electromagnetic Research, 2007, vol. 74, p. 47-56. DOI: 10.2528/PIER07041904

[9] HAZDRA, P., CAPEK, M., MASEK, M., LONSKY, T. An introduction to the source concept for antennas. Radioengineering, 2016, vol. 25, no. 1, p. 12-17. DOI: 10.13164/re.2016.0012

[10] MathWorks. [Online] Cited 2017-01-20. Available at: www.mathworks.com

[11] HAZDRA, P., CAPEK, M., EICHLER, J., MAZANEK, M. The radiation $\mathrm{Q}$-factor of a horizontal $\lambda / 2$ dipole above ground plane. IEEE Antennas and Wireless Propagation Letters, 2014, vol. 13, p. 1073-1075. DOI: 10.1109/LAWP.2014.2329421

\section{About the Authors ...}

Michal CERVENY received his M.Sc. from the Czech Technical University (CTU) in Prague in 2014. He is working towards his Ph.D. focused on the behavior of metasurfaces for RCS limitation.

Pavel HAZDRA received his M.Sc. and Ph.D. from the Czech Technical University (CTU) in Prague in 2003 and 2009, respectively and was appointed an Associate Professor in 2012. His research interests include antenna and EM theory, computational electromagnetics and special primefeed antenna structures. 